\title{
Un nuevo xenófito del género Baccharis L. (Asteraceae) para el centro de la Península Ibérica
}

\author{
Antonio Galán de Mera ${ }^{1}$ Eliana Linares Perea ${ }^{2} \&$ José Alfredo Vicente Orellana ${ }^{1}$ \\ ${ }^{1}$ Laboratorio de Botánica, Facultad de Farmacia, Universidad CEU San Pablo, CEU Universities, apartado 67, 28660 \\ Boadilla del Monte, Madrid, España \\ Estudios Fitogeográficos del Perú, Sánchez Cerro 219, Manuel Prado, Paucarpata, Arequipa, Perú
}

\section{Correspondencia}

A. Galán de Mera

e-mail: agalmer@ceu.es

Recibido: 29 enero 2021

Aceptado: 31 mayo 2021

Publicado on-line: 3 junio 2021

Editado por: Andrés V. Pérez Latorre

\begin{abstract}
Resumen
Se indica la presencia de Baccharis pilularis DC. subsp. consanguinea (DC.) C.B. Wolf como posible nueva planta invasora en la península lbérica.
\end{abstract}

Palabras clave: Baccharis, bioclimatología, clave de especies, Península Ibérica, xenófitos.

\begin{abstract}
A new xenophyte of the genus Baccharis L. (Asteraceae) for the central Iberian Peninsula

Baccharis pilularis DC. subsp. consanguinea (DC.) C.B. Wolf is reported in the Iberian peninsula as a possible new invasive plant.
\end{abstract}

Key words: Baccharis, bioclimatology, species key, Iberian peninsula, xenophytes.
Baccharis L. es un género americano que reúne a unas 400 especies sobre todo de arbustos y subarbustos, dioicos, con hojas de ovado-elípticas a cuneadas, con frecuencia lustrosas, y capítulos femeninos o funcionalmente masculinos (Hellwig, 1990; Giuliano \& Plos, 2014; Sundberg \& Bogler, 2021). En Europa se han detectado varias especies comportándose como xenófitos: Baccharis articulata (Lam.) Pers. y B. glutinosa Pers. (= B. pingraea DC.) en Huelva (Bartoli et al., 2007), B. salicifolia (Ruiz \& Pav.) Pers. en Barcelona (Gómez-Bellver et al., 2016), y B. spicata (Lam.) Baill. en los alrededores de Oporto (Verloove et al., 2017). Pero de todas ellas la más extendida es $B$. halimifolia $L$., con citas en Bélgica, Francia, Georgia, Gran Bretaña, Holanda, y España con varias poblaciones en el norte, en Asturias, País Vasco, Galicia y Santander (Sanz Elorza et al., 2004; Euro+Med, 2006-2021; Anthos, 2021), habiéndose introducido también en Australia y Nueva Zelanda (CABI, 2021). Gassó et al. (2012) indican su distribución potencial en la provincia de Madrid, que igualmente se recoge en el Catálogo Español de Especies Exóticas Invasoras (MITECO, 2021) aunque no hemos encontrado testigo de herbario que lo confirme (GIBF-ES, 2021).

Aportamos una cita de B. pilularis DC. subsp. consanguinea (DC.) C.B. Wolf como nueva para Europa y España, cuyos pliegos de herbario se encuentran depositados en el herbario de la Universidad CEU San Pablo (USP):
Baccharis pilularis DC. subsp. consanguinea (DC.) C.B. Wolf in Occas. Pap. Rancho Santa Ana Bot. Gard. 1(1): 21 (1935)

ESPAÑA. Madrid: Las Rozas, El Cantizal, 40³1'08.49"'N-356'13.14"'O, 625 m, 29-IX-2020, E. Linares, J.A. Vicente \& A. Galán 4797, ejemplares femeninos (USP 3501); E. Linares, J.A. Vicente \& A. Galán 4796, ejemplares masculinos (USP 3502); $E$. Linares, J.A. Vicente \& A. Galán 4798, ejemplar masculino (USP 3503).

De acuerdo con nuestras observaciones en los herbarios MO y US, $B$. pilularis se asemeja a $B$. halimifolia pues ambas tienen aspecto de escobón, pero ésta última muestra los capítulos pedunculados formando inflorescencias paniculiformes (Morales, 2019), frente a los capítulos sentados de $B$. pilularis (Sundberg \& Bogler, 2021) que forman inflorescencias espiciformes (Figura 1). Proponemos la siguiente clave para separar las especies presentes en la península Ibérica:

1. Ramas áfilas, aladas................ . B. articulata 1. Ramas con hojas, frecuentemente acostilladas....2 2. Hojas de ovado-elípticas a lineares (especies Sudamericanas) $\ldots \ldots \ldots \ldots \ldots \ldots \ldots \ldots \ldots \ldots \ldots \ldots \ldots . \ldots . \ldots \ldots$

2. Hojas cuneadas (especies Norteamericanas) .......5

3. Hierbas con hojas aserradas.........2. B. glutinosa

3. Arbustos o subarbustos con las hojas enteras, aserradas en parte 0 dentadas........................ 4 4. Todas las hojas alternas, enteras o aserradas en 
los $2 / 3$ superiores.

\section{B. salicifolia} 4. Hojas inferiores opuestas, irregularmente dentadas en toda su longitud. 4. B. spicata 5. Capítulos pedunculados con las brácteas del involucro con pequeños pelos glandulíferos, formando inflorescencias paniculiformes escamosas......................................... 5. Capítulos sentados con las brácteas del involucro glabras, frecuentemente en glomérulos de 2-4 capítulos, formando inflorescencias espiciformes, con papilas y glándulas punteadas..

6. B. pilularis

Baccharis pilularis procede de América del Norte reuniendo a dos subespecies: $B$. pilularis subsp. pilularis, de la costa californiana, con las ramas prostradas; y $B$. pilularis subsp. consanguinea, de áreas interiores de California, Nuevo México y Oregón, y Baja California en México (Sundberg \& Bogler, 2021), con las ramas erguidas.
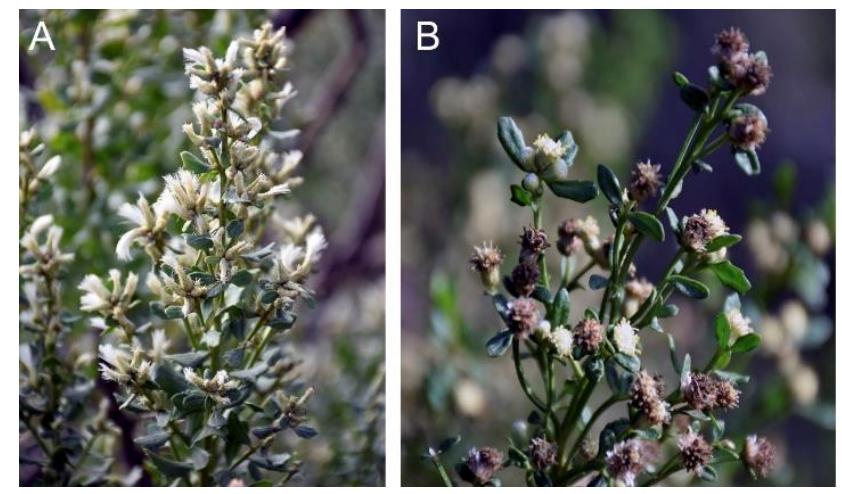

Figura 1. Ramas de un arbusto femenino (A) y otro masculino (B) de Baccharis pilularis subsp. consanguinea en Madrid (España).

Figure 1. Branches of a female bush (A) and a male bush (B) of Baccharis pilularis subsp. consanguinea in Madrid (Spain).

Los individuos de las tres poblaciones encontradas en el centro de España parecen comportarse como metáfitos (Kornas, 1990) y xenófitos neoindigenófitos (holoagriófitos) (Pyšek et al., 2004), introducidos casualmente a partir del jardín construido en una rotonda. La población más grande consta de más de 100 plantas (la mayor parte de ellas son todavía estériles, pero hemos contado unas 3 femeninas fructificadas y 3 masculinas bien desarrolladas, de más de $1 \mathrm{~m}$ ) que viven en el talud de una carretera donde los aquenios se dispersan con el viento generado por los automóviles; otra población progresa en un retamar muy alterado, y está formada por un arbusto femenino y dos masculinos; y una tercera población, formada por varias plantas masculinas, se encuentra junto al muro de una casa. La distribución de los arbustos y la dispersión de los aquenios desde el lugar ajardinado, sigue la dirección $\mathrm{O}-\mathrm{SO}$ de los vientos dominantes en el $\mathrm{NO}$ de la provincia de Madrid (Kaufmann, 2021).

En su lugar de origen, $B$. pilularis subsp. consanguinea tiene preferencia por lugares abiertos y áreas alteradas con escombros y taludes (CABI,
2020), ocupando en Estados Unidos áreas de los termotipos termo- y mesomediterráneo y ombrotipos semiárido y seco (Cress et al., 2009). En nuestro territorio crece asociada a una arbusteda mesomediterránea seca abierta de Cytiso scopariiRetametum sphaerocarpae Rivas-Martínez ex V. Fuente 1986, sobre los suelos arenosos de la provincia biogeográfica Mediterránea Ibérica Occidental, sector Guadarrámico, distrito Altomatritense (Rivas-Martínez, 2007), junto a Crataegus monogyna Jacq., Pinus pinea L., Quercus rotundifolia Lam. y Retama sphaerocarpa Boiss.

Todo ello indica que la similitud habitacional de $B$. pilularis subsp. consanguinea entre las localidades originarias y las alóctonas en el centro ibérico podría predecir su potencialidad invasora, teniendo en cuenta la proliferación de los individuos en los últimos años, y el comportamiento de otras especies del género, como B. halimifolia (Sanz Elorza et al., 2004) y $B$. spicata, en otros espacios de la península (Verloove et al., 2017). Naturalmente estos resultados avisan de la peligrosidad del cultivo de ciertas especies alóctonas, más aún cuando proceden de áreas del mundo similares climáticamente, por lo que se recomienda el seguimiento cartográfico y erradicación de los individuos de $B$. pilularis subsp. consanguinea, seguido del cultivo de otras especies autóctonas en las zonas ajardinadas del oeste de la provincia de Madrid, al menos en las próximas a las carreteras.

\section{Bibliografía}

Anthos. (2021). Sistema de información sobre las plantas de España. Real Jardín Botánico, CSICFundación Biodiversidad. Recurso electrónico en www.anthos.es. Consulta realizada el 12/01/2021.

Bartoli, A., Sánchez Gullón, E., Weickert, P. \& Tortosa, R.D. (2007). Plantas americanas nuevas para la flora adventicia del sur de España. Acta Botanica Malacitana, 32, 276-282. https://doi.org/10.24310/abm.v32i0.7058

CABI. (2021). Invasive Species Compendium. Wallingford, UK: CAB International. Recurso electrónico en www.cabi.org/isc. Consulta realizada el 08/01/2021.

Cress, J.J., Roger, S., Patrick, C. \& Warner, H. (2009). Terrestrial Ecosystems-Isobioclimates of the conterminous United States: U.S. Geological Survey Scientific Investigations Map 3084, scale 1: 5,000,000. Denver, CO: Denver Publishing Service Center.

Euro+Med. (2006-2021). Euro+Med PlantBase-the information resource for Euro-Mediterranean plant diversity. Palermo: Università degli Studi di Palermo. Recurso electrónico en http://ww2.bgbm.org/EuroPlusMed/. Consulta realizada el 11/01/2021.

Gassó, N., Thuiller, W., Pino, J. \& Vilà, M. (2012). Potential distribution range of invasive plant species in Spain. NeoBiota, 12, 25-40. 
https://doi.org/10.3897/neobiota.12.2341.app2

GBIF-ES. [2021]. Nodo Nacional de Información en Biodiversidad. Madrid: Ministerio de Ciencia e Innovación. Recurso electrónico en

https://www.gbif.es/. Consulta realizada el 29/05/2021.

Giuliano, D.A. \& Plos, A. (2014). Baccharis L. In F.O. Zuloaga, M.J. Belgrano \& A.M. Anton (Eds.), Flora Argentina, vol. 7, tomo I (pp. 43-123). Buenos Aires: Instituto de Botánica Darwinion.

Gómez-Bellver, C., Álvarez, H. \& Sáez, L. (2016). New contributions to the knowledge of the alien flora of the Barcelona province (Catalonia, Spain). Orsis, 30, 167-189.

https://doi.org/10.5565/rev/orsis.36

Kaufmann, J. (2021). Windfinder. Kiel: Windfinder.com GmbH \& Co. KG. Recurso electrónico en https://es.windfinder.com/. Consulta realizada el 21/01/2021.

Kornas, J. (1990). Plant invasions in Central Europe: historical and ecological aspects. In F. Di Castri, A.J. Hansen \& M. Debussche (Eds.), Biological Invasions in Europa and the Mediterranean Basin (pp. 19-36). Dordrecht, NL: Kluwer Academic Publishers.

Hellwig, F.H. (1990). Die Gattung Baccharis L. (Compositae-Asteraceae) in Chile. Mitteilungen aus der Botanischen Staatssammlung München, 29, 1-456.

MITECO (2021). Catálogo Español de Especies Exóticas Invasoras-Flora. Madrid: Ministerio para la Transición Ecológica y el Reto Demográfico.
Recurso electrónico en

https://www.miteco.gob.es/es/biodiversidad/tema s/conservacion-de-especies/especies-exoticasinvasoras/ce_eei_flora.aspx. Consulta realizada el 29/05/2021.

Morales, R. (2019). Baccharis L. In S. Castroviejo (Coord.), Flora iberica, vol. XVI (III) (pp. 20402043). Madrid: CSIC.

Pyšek, P., Richardson, D.M., Rejmánek, M., Webster, G.L., Williamson, M. \& Kirschner, J. (2004). Alien plants in checklists and floras: towards better communication between taxonomists and ecologists. Taxon, 53(1), 131-143. https://doi.org/10.2307/4135498

Rivas-Martínez, S. (2007). Mapa de series, geoseries y geopermaseries de vegetación de España. Itinera Geobotanica, 17, 5-436.

Sanz Elorza, M., Dana Sánchez, E.D. \& Sobrino Vesperinas, E. (2004). Atlas de las plantas alóctonas invasoras en España. Madrid: Ministerio del Medio Ambiente.

Sundberg, S.D. \& Bogler, D.J. (2021). Baccharis L. Flora of North America vol. 20, Sant Louis, MO: Missouri Botanical Garden. Recurso electrónico en www.efloras.org. Consulta realizada el $11 / 01 / 2021$

Verloove, F., Dana, E.D. \& Alves, P. (2017). Baccharis spicata (Asteraceae), a new potentially invasive species to Europe. Plant Biosystems, 152(3), 416-426.

https://doi.org/10.1080/11263504.2017.1303001 